\title{
Forming a primordial star in a relic HII region
}

\author{
Brian W. O'Shea ${ }^{1}$, Tom Abel ${ }^{2}$, Dan Whalen ${ }^{1}$ \& Michael L. Norman ${ }^{3}$ \\ 1 Theoretical Astrophysics Group, Los Alamos National Laboratory \\ email: bwoshea@lanl.gov \\ ${ }^{2}$ Kavli Institute for Particle Astrophysics and Cosmology, Stanford University \\ ${ }^{3}$ Center for Astrophysics and Space Sciences, University of California in San Diego
}

\begin{abstract}
We present results from an Eulerian adaptive mesh refinement simulation demonstrating the formation of a primordial star within an HII region produced by an earlier massive star. Despite the higher temperatures of the ionized gas, this second star formed within 23 million years of its neighbor's death. The enhanced electron fraction within the HII region catalyzes rapid molecular hydrogen formation, leading to enhanced cooling in the preprocessed halo. This "second generation" primordial protostar has a much lower accretion rate than its predecessor, due in part to the higher angular momentum of the halo in which it forms. This situation may allow binaries or multiple systems of low-mass stars to form. The result discussed here is described in more detail in O'Shea et al. (2005).
\end{abstract}

Keywords. Stars: formation, HII regions, large-scale structure of universe

\section{Introduction}

Calculations performed by Abel, Bryan, \& Norman (2002) show that rapid accretion rates driven by molecular hydrogen cooling cause the formation of solitary massive protostars in the range of $30-300 M_{\odot}$ in minihalos with masses of $10^{5}-10^{6} M_{\odot}$ at $z>20$. As shown by Whalen et al. (2004), the copious UV emission from these stars is capable of ejecting the majority of baryons from the halo at rates of up to 10 times the halo's escape velocity. After this star dies, cooling and recombination are out of equilibrium, resulting in a significant electron fraction even at low temperatures and late times. Calculations by Heger et al. (2003) predict that stars in this mass range have two possible fates complete destruction in a "pair instability" supernova or direct collapse into a black hole. Assuming the latter case, in this paper we present simulations that demonstrate a star can form in the relic HII region of an earlier Pop III star and we estimate the mass of this star (see O'Shea et al. (2005) for more details).

\section{Problem setup and results}

We carried out these simulations using Enzo, a publicly-available Eulerian adaptive mesh refinement (AMR) hydrodynamics + N-body code (O'Shea et al. (2004)). We use a periodic simulation volume $300 \mathrm{~h}^{-1} \mathrm{kpc}$ (comoving) on a side with a fiducial $\Lambda \mathrm{CDM}$ cosmology. We initialize the calculation at $z=99$ using a set of static nested subgrids centered on the most massive halo and evolve this volume forward to the collapse of the first protostar, following N-body dark matter dynamics as well as hydrodynamics with nonequilibrium primordial chemistry and radiative cooling. 
We then use a ray-tracing code to determine the extent of the HII region this Pop III star would create by the end of its main sequence lifetime, imposing the sphericallysymmetric hydrodynamical profiles from Whalen et al. (2004) along each line of sight from the star within the halo but modifying only temperatures and ionization fractions beyond the halo. After inserting this HII region into the simulation volume we continue our calculation until the formation of the second protostar, which occurs approximately 23 million years later. The formation of this protostar and its properties are insensitive to the problem setup.

The second primordial protostar forms in a neighboring minihalo, approximately 265 proper parsecs from the first star. The halo in which this object forms was completely ionized by the first star, but due to its relatively high density the center of this halo cools very quickly. Rapid formation of molecular hydrogen is catalyzed by the high electron fraction. Comparison of the mass accretion rate of the two protostars reveals that the accretion rate onto the second is more than an order of magnitude lower than onto the first.

Plausible estimates of the main-sequence masses of the two protostars suggest that the first will evolve into a star with mass $\mathrm{M}_{*} \sim 30-300 \mathrm{M}_{\odot}$ while the second may form a star of much lower mass, $\mathrm{M}_{*} \sim 5-20 \mathrm{M}_{\odot}$. This lower mass range is due in part to the high angular momentum of the second halo compared to the first $\left(\lambda_{\text {gas }}=0.0275\right.$ for the first halo, $\lambda=0.1079$ for the second), which causes a rotationally supported disk containing $\sim 100 \mathrm{M}_{\odot}$ of gas to develop around the second protostar.

\section{Discussion and Conclusions}

These simulations directly address the formation of individual primordial stars in an HII region produced by an earlier massive star. We show that it is possible to form stars in a relic HII region, and that Population III stars can be formed with significantly lower masses than previously thought (e.g. Abel, Bryan \& Norman (2002). Further study is required to determine if the results of these calculations are generally true or are specific to our particular problem setup. Low-mass primordial stars or binaries and multiple star systems would have very different lifetimes, nucleosynthetic signatures, and observabilities than very massive first generation stars, with serious consequences for the formation of later generations of stars. The large size of early HII regions could make this scenario more common than that of stars forming in unprocessed halos.

\section{Acknowledgements}

This work supported in part by NASA grant NAG5-12140, NSF grant AST-0307690 and by NSF CAREER award AST-0239709. The simulations were performed at SDSC and NCSA with computing time provided by NRAC allocation MCA98N020. BWO and DW have been funded in part under the auspices of the U.S. Dept. of Energy, and supported by its contract W-7405-ENG-36 to Los Alamos National Laboratory.

\section{References}

Abel, T., Bryan, G. L., \& Norman, M. L. 2002, Science 295, 93

Heger, A., Fryer, C.L., Woosley, S.E., Langer, N. \& Hartmann, D.H. 2003, ApJ 591, 288

O'Shea, B.W., Bryan, G., Bordner, J., Norman, Michael L., Abel, T., Harkness, R. \& Kritsuk, A. 2004, in Adaptive Mesh Refinement - Theory and Applications Eds. T. Plewa, T. Linde \& V. G. Weirs, Springer Lecture Notes in Computational Science and Engineering O'Shea, B.W., Abel, T., Whalen, D. \& Norman, M.L. 2005, ApJ in press, astro-ph/0503330 Whalen, D., Abel, T., \& Norman, M. L. 2004, ApJ 610, 14 


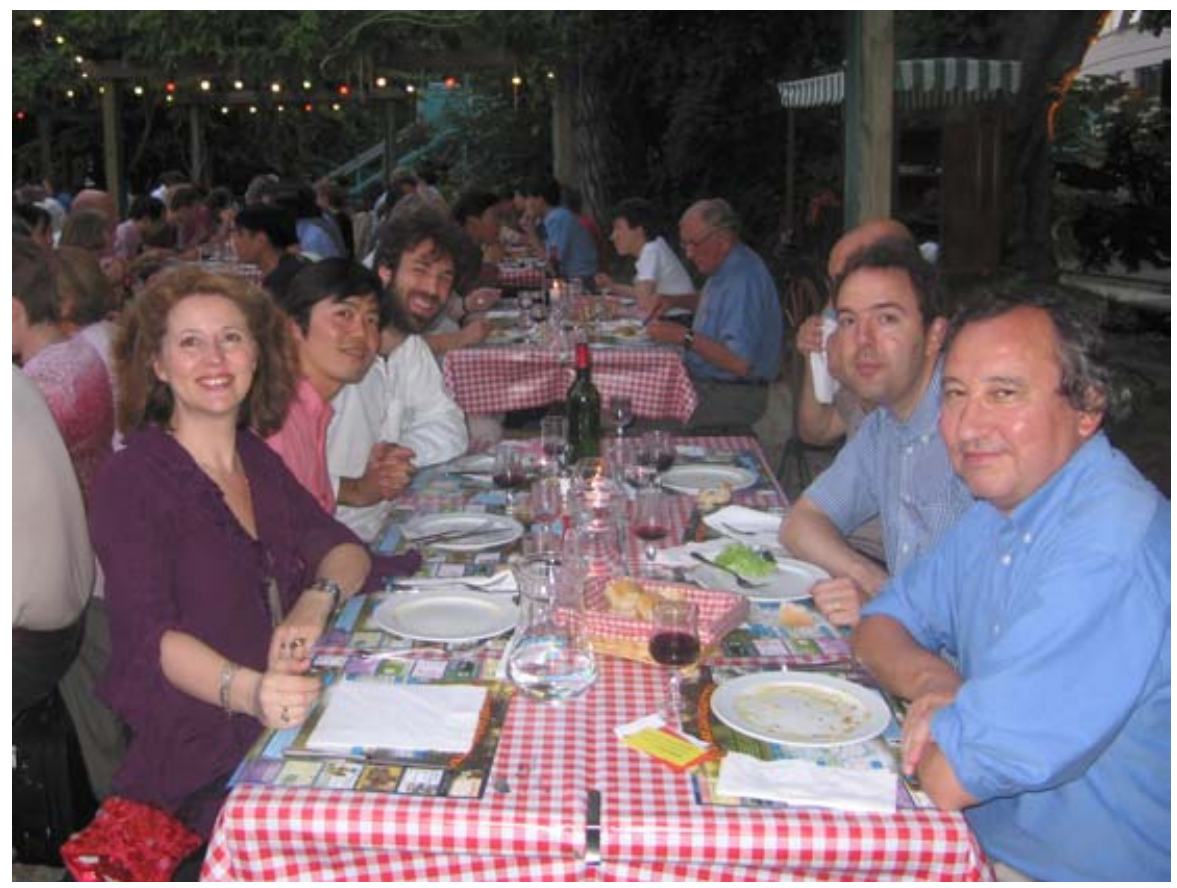

Elisabeth Vangioni-Flam, Susumu Inoue and Emmanuel Rollinde, facing Frederic Daigne and Alain Coc, at the conference dinner table.

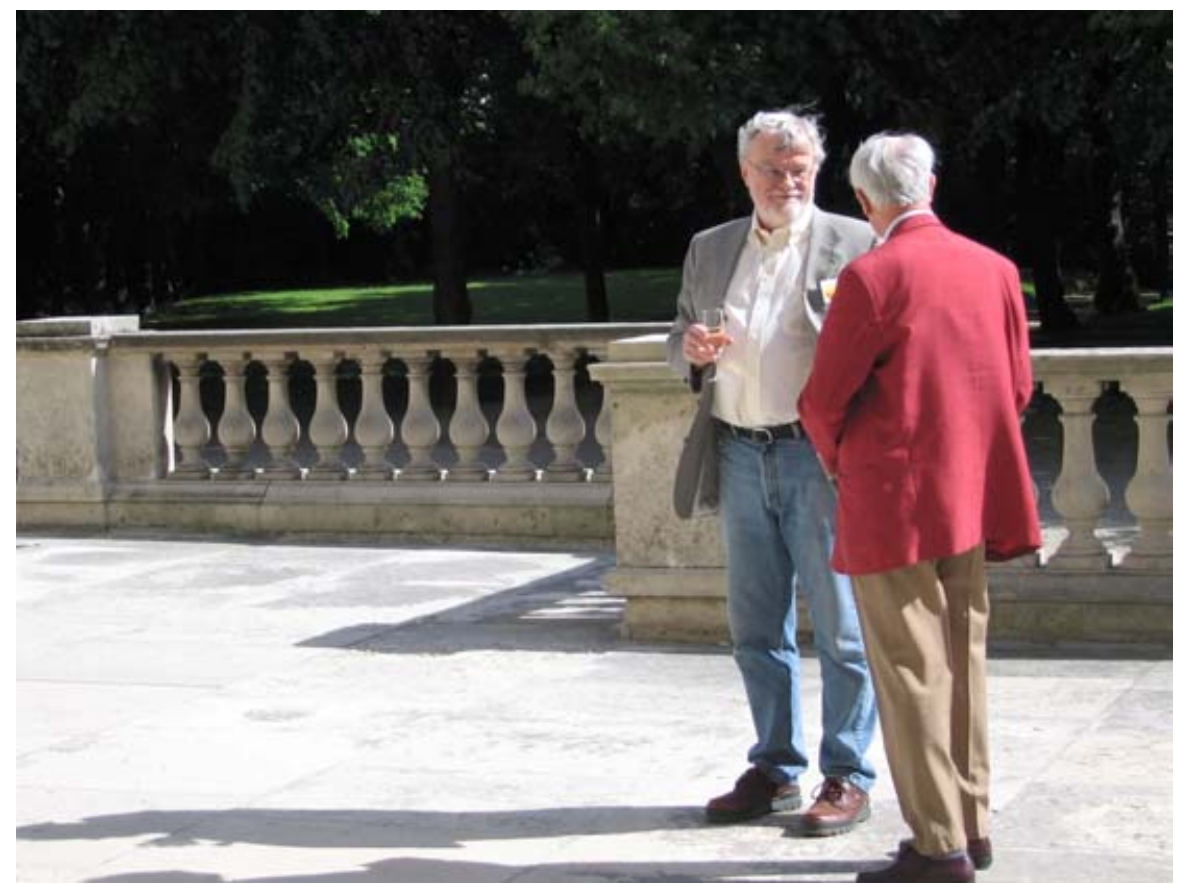

David Arnett and Roger Cayrel, blown in the wind, on the Paris Observatory terrace during the welcome reception. 


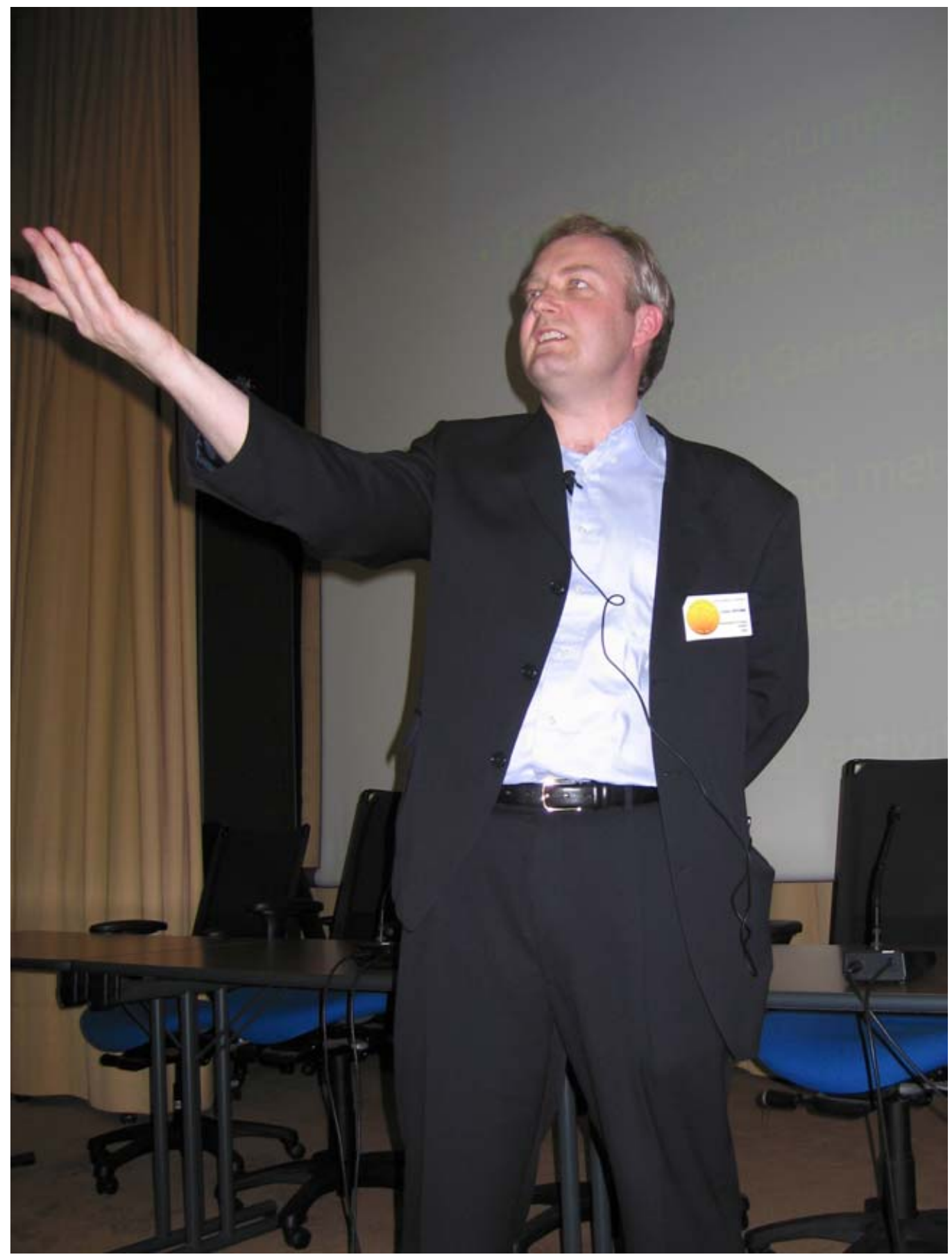

Volker Bromm, weighing the First Stars to form in the Universe. 